\title{
Kaposi's sarcoma-associated herpesvirus infection and Kaposi's sarcoma in Brazil
}

S. Ramos-da-Silva ${ }^{1}$, D. Elgui-de-Oliveira ${ }^{1,2}$, L. Borges ${ }^{1}$ and C.E. Bacchi²

\author{
'Departamento de Patologia, Faculdade de Medicina de Botucatu, \\ Universidade Estatual Paulista, Botucatu, SP, Brasil \\ ${ }^{2}$ Consultoria em Patologia, Botucatu, SP, Brasil
}

\begin{abstract}
Correspondence
C.E. Bacchi

Rua Major Leônidas Cardoso, 739

18602-010 Botucatu, SP

Brasil

E-mail:

bacchi@consultoriapatologia.com.br

Research supported by FAPESP (Nos. 2000/11130-2 and 2000/12248-7).
\end{abstract}

Received December 17, 2004 Accepted September 30, 2005

\begin{abstract}
Kaposi's sarcoma (KS) became a critical health issue with the emergence of acquired immunodeficiency syndrome (AIDS) in the 1980s. Four clinical-epidemiological forms of KS have been described: classical KS, endemic KS, iatrogenic KS, and AIDS-associated KS. In 1994, Kaposi's sarcoma-associated herpesvirus (KSHV) or human herpesvirus type 8 was identified by Chang and colleagues, and has been detected worldwide at frequencies ranging from 80 to $100 \%$. The aim of the present study was to evaluate the frequency of KSHV infection in KS lesions from HIV-positive and HIV-negative patients in Brazil, as well as to review the current knowledge about KS transmission and detection. For these purposes, DNA from 51 cases of KS was assessed by PCR: 20 (39.2\%) cases of classical KS, 29 (56.9\%) of AIDS-associated KS and 2 (3.9\%) of iatrogenic KS. Most patients were males $(7.5: 1, \mathrm{M} / \mathrm{F})$, and mean age was 47.9 years $(\mathrm{SD}=$ \pm 18.7 years). As expected, HIV-positive KS patients were younger than patients with classical KS. On the other hand, patients with AIDS-associated KS have early lesions (patch and plaque) compared to classical KS patients (predominantly nodular lesions). This is assumed to be the result of the early diagnose of KS in the HIVpositive setting. KSHV infection was detected by PCR in almost all cases $(48 / 51 ; 94.1 \%)$, irrespectively of the clinical-epidemiological form of KS. These results show that KSHV is associated with all forms of KS in Brazilian patients, a fact that supports the role of this virus in KS pathogenesis.
\end{abstract}

\section{Introduction}

In 1872, Kaposi's sarcoma (KS) was described by Moritz Kaposi (1) as a rare and indolent angioproliferative neoplasm, mainly present as a skin lesion (2). Since the 1980's, the disease has become a critical health issue with the emergence of the AIDS epidemic
Key words

- Kaposi's sarcoma

- Human herpesvirus type 8

- Kaposi's sarcoma-associated herpesvirus

- Polymerase chain reaction

- Viral carcinogenesis

- Brazil
(2). The classical form of $\mathrm{KS}$ is characterized by solitary plaques or nodules in the lower extremities of elderly men from Eastern Europe and the Mediterranean region (3). Three other clinical-epidemiological forms are known: endemic KS, affecting children and young men in Central Africa (4); iatrogenic KS, observed in some patients under immu- 
nosuppressive therapy (5), and AIDS-associated $\mathrm{KS}$, an aggressive form of $\mathrm{KS}$ that occurs mainly in homosexual and bisexual men (6).

In 1994, Chang and co-workers (7) identified unique herpesvirus DNA sequences in KS lesions from HIV-positive patients. The new virus, Kaposi's sarcoma-associated herpesvirus (KSHV) or human herpesvirus type 8 (HHV-8), is also demonstrable in tumors other than KS (8-11). The viral genome encodes several products that share structural and functional homology with human proteins that have pivotal role in cell proliferation, which may explain the viral contribution to a malignant transformation (1218).

The worldwide frequency of KSHV detected in KS ranges from 80 to $100 \%$. Frequently used methods to assess viral infection are in situ hybridization, serologic analysis and polymerase chain reaction (PCR) (19-21), the latter being the most sensitive. Using PCR, Moore and Chang (22) detected $\mathrm{KSHV}$ in 6/6 (100\%) classical KS cases, in 4/4 (100\%) HIV-negative homosexual patients, and 10/11 (91\%) AIDS-associated $\mathrm{KS}$ cases. In a similar study, Dictor et al. (23) found the virus in all $14 \mathrm{KS}$ cases from HIVpositive patients, as well as in 35/40 (88\%) classic KS cases.

Data about KSHV infection in KS occurring in Brazilian patients are scarce (24-27). The objective of the present study was to identify the viral genome in KS lesions from HIV-positive and HIV-negative patients in Brazil.

\section{Material and Methods}

The study was approved by the Research Ethics Committee of the Botucatu Medical School, São Paulo State University (UNESP). Tissue biopsies from $51 \mathrm{KS}$ lesions were obtained from the files of the Department of Pathology, Botucatu Medical School, and from private Surgical Pathology laborato- ries in São Paulo State, Brazil. Based on clinical and histopathological data, the cases were divided as follows: $20(39.2 \%)$ cases of classical KS, 29 (56.9\%) of AIDS-associated KS and 2 (3.9\%) of iatrogenic KS. Skin biopsies with normal histology $(\mathrm{N}=8)$ were used as negative controls for KSHV infection.

The lesions were further classified as patch, plaque or nodules according to the stage of the disease. Early stages of KS are characterized by purple patches on the skin, which are histologically characterized by neoplastic cells scattered in the dermis. In the plaque stage, confluent groups of neoplastic cells are observed, as well as increased neovascularization and hemosiderin deposition. The nodular stage consists of tumors composed of high density of spindleshaped cells and other components, such as endothelial cells, inflammatory infiltrate and erythrocytes.

At least two 25- $\mu \mathrm{m}$ sections were obtained from formalin-fixed paraffin-embedded tissues for DNA extraction. Briefly, sections were deparaffinized with xylene (10 $\min$ at $64^{\circ} \mathrm{C}, 10 \mathrm{~min}$ at room temperature with shaking, and again $10 \mathrm{~min}$ at $64^{\circ} \mathrm{C}$ ). Next, the samples were centrifuged for 5 $\min$ at 15,800 $g$ and xylene was removed and this sequence was repeated twice; the tissues were then dehydrated with ethanol and centrifuged for $10 \mathrm{~min}$ at $15,800 \mathrm{~g}$. Ethanol was removed and these steps were repeated once. Subsequently, tissue sections were incubated overnight with proteinase $\mathrm{K}(400 \mathrm{ng} / \mu \mathrm{L}$ in $10 \mathrm{mM}$ Tris, $1 \mathrm{mM}$ EDTA, 0.5\% Tween 20 buffer) at $56^{\circ} \mathrm{C}$.

After enzyme inactivation by heat $\left(94^{\circ} \mathrm{C}\right.$ for $10 \mathrm{~min}$ ), DNA purification was performed by adding $100 \mu \mathrm{L} 5 \mathrm{mM} \mathrm{NaCl}$ plus $100 \mu \mathrm{L}$ of the $\mathrm{CTAB} / \mathrm{NaCl}$ solution $(0.87 \mathrm{M} \mathrm{NaCl}, 0.34$ $\mathrm{M}$ CTAB) to the samples. After $10 \mathrm{~min}$ of incubation at $65^{\circ} \mathrm{C}$, the samples were treated with 1:24 chloroform/isoamilic alcohol and centrifuged for $5 \mathrm{~min}$ at $15,800 \mathrm{~g}$. The upper phase was transferred to a new tube with 
ethanol, and kept at $-20^{\circ} \mathrm{C}$ for $10 \mathrm{~min}$. After another centrifugation at $15,800 \mathrm{~g}$, the precipitated DNA was treated with $70 \%$ ethanol at room temperature, and the pellet was resuspended in $50 \mu \mathrm{L}$ TE solution $(10 \mathrm{mM}$ Tris-Cl, $\mathrm{pH} 7.5,1 \mathrm{mM}$ EDTA). At this point, the quantity and quality of DNA were determined by UV-spectrophotometry and agarose-gel electrophoresis, respectively. Samples in which DNA concentration was higher than $0.4 \mu \mathrm{g} / \mu \mathrm{L}$ were diluted to $0.1 \mu \mathrm{g} / \mu \mathrm{L}$ to prevent inhibition of the PCR.

KSHV detection by PCR was initially carried out with KS330 primers (7). The samples without amplification in this first PCR assay were used in ORF26-directed nested-PCR (28). Doubtful cases were analyzed for amplification of viral ORF72 segment, which encodes a viral capsid protein (28). Samples negative for all PCR assays were further evaluated for amplification of the $B$-chain human globin gene with $\mathrm{PCO}_{3}+/$ $\mathrm{PCO}_{4}+$ primers (29). $\mathrm{PCR}$ components were: buffer (28 mM Tris-Cl, pH 8.4, $70 \mathrm{mM} \mathrm{KCl}$, $3.0 \mathrm{mM} \mathrm{MgCl}_{2}$ ), $0.2 \mathrm{mM}$ each dNTP, $1.25 \mathrm{U}$ Taq DNA polymerase (Invitrogen, Carlsbad, CA, USA), and $0.5 \mu \mathrm{M}$ of upstream and downstream primers. The PCR cycling profile was $94^{\circ} \mathrm{C}$ for $5 \min (1 \mathrm{X}) ; 94^{\circ} \mathrm{C}$ for $30 \mathrm{~s}$, AT for $30 \mathrm{~s}, 72^{\circ} \mathrm{C}$ for $1 \mathrm{~min}(35 \mathrm{X}) ; 72^{\circ} \mathrm{C}$ for $7 \mathrm{~min}(1 \mathrm{X})$, where AT is the annealing temperature for each primer pair: $57^{\circ} \mathrm{C}$ for KS330 primers and $55^{\circ} \mathrm{C}$ for both ORF26in and ORF72 primers. The PCR product was analyzed in $2 \%$ agarose gel stained with ethidium bromide. The expected amplicon sizes were 233 bp for KS330 primers, and $138 \mathrm{bp}$ for both ORF26in and ORF72 primers.

Besides KS tissues, 8 normal skin biopsies were checked for KSHV infection by PCR using the same procedure as described above (namely KS330 PCR, ORF26 nestedPCR and ORF72). Additionally, PCR products of two randomly chosen KSHV-positive KS samples from each PCR assay were submitted to automated nucleic acid sequencing to assure the specificity of the molecular protocol used to detect KSHV infection in paraffin-embedded tissues.

\section{Results}

Using KS330 primers KSHV infection was detected in $31 / 51(60.8 \%) \mathrm{KS}$ cases. Using ORF26-directed nested-PCR (ORF26in primers), $14 / 20$ (70\%) of the originally negative cases proved to be KSHV-positive, while 3 of the $6(50 \%)$ remaining cases had demonstrable viral ORF72 amplification. Thus, $94.1 \%$ of all KS cases were KSHV-positive. The KSHV genome was detected in all KS cases regardless of KS type. Normal skin biopsies, used as negative controls, were consistently negative for KSHV infection and suitable for amplification of a 123-bp Bglobin gene segment. The specificity of KS330, ORF26in, and ORF72 PCR assays for KSHV detection was confirmed by automated sequencing of randomly chosen PCR products of KSHV-positive KS samples, as shown in Figure 1.

Patients with AIDS-associated KS were younger than those with classical KS. Regarding the stage of the disease, patients with classical KS predominantly presented nodular lesions, while HIV-positive patients had the early stages of KS (patch and plaque). These data are probably due to early identification of KS in HIV-positive patients, who often exhibited multiple lesions, facilitating clinical detection. Table 1 summarizes the available data on the KS cases evaluated, including clinical information and the KSHV infection status.

\section{Discussion}

Despite the increasing number of studies on KSHV and human neoplasia, to date the role of viral infection in the pathogenesis of KS has not been completely elucidated. It is well known that the KSHV genome encodes products that share homology with human cell proteins. For instance, viral bcl-2 (en- 
A

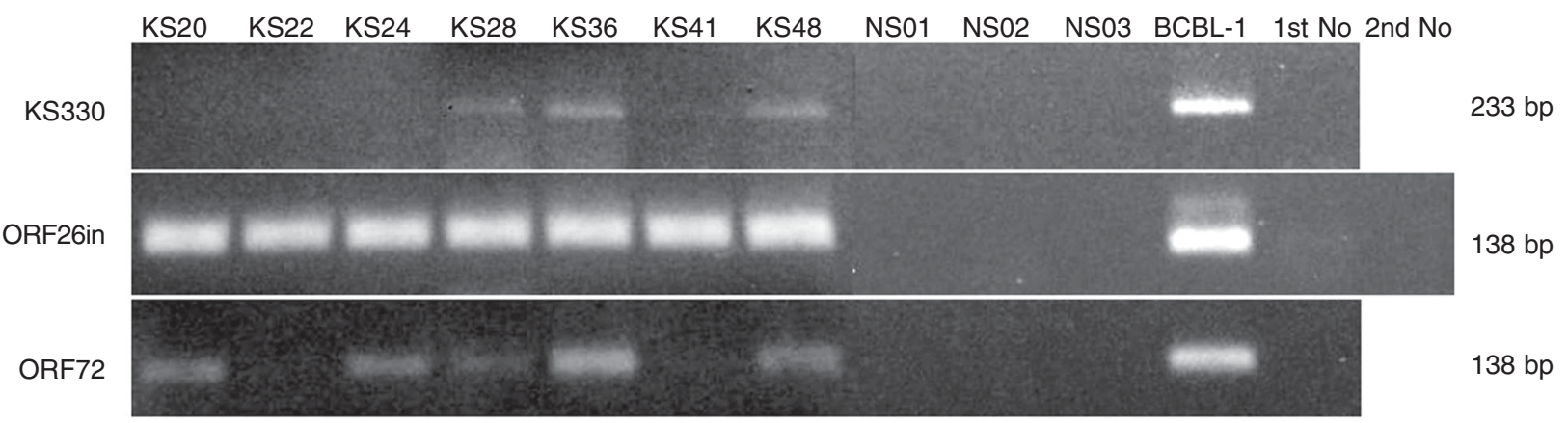

B

$\mathrm{U} 18551$ *

$1 \mathrm{KS} 330 . \mathbf{S}$
-AGCCGAAAGGATTC-CACCATTGTGCTCGAATCCAAC-GGATTTGACCCCGTGTTCC-CCATGGTCGTGC-CGCAGCAACTGGGGCACGCTATTC-TGC

$\mathrm{BCBL}-1$

KS36

KS4 8

KS20

KS22

KS2 4

101

$\mathrm{U} 18551$ *

BCBL-1

KS36

KS4 8

KS20

KS2 2

KS24

U18551 *

BCBL-1

KS3 6

KS4 8

KS2 0

KS2 2

KS24

C

U 40667 *

KS2 4

KS36

KS 48

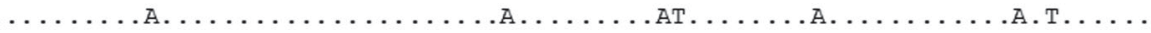
.

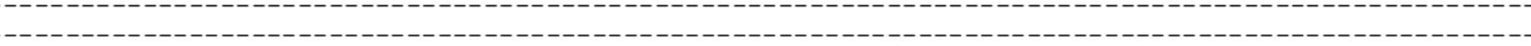
AGCAGCTGTTGGTGTACCACATCTACTCCAAAATATC-GGCC---GGGGCCCCGGATGATGT---AAATATG---GCGGAACTTGATCTATATACCACCA

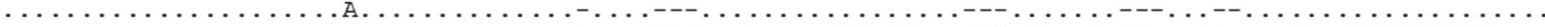

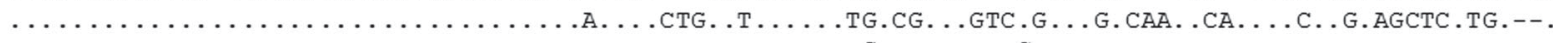

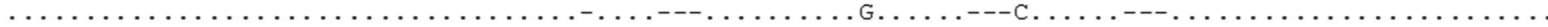

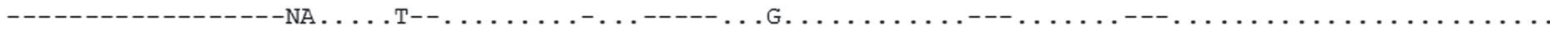

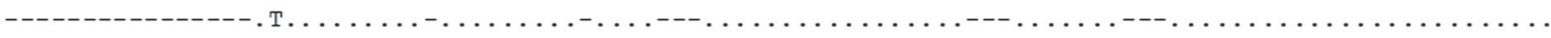

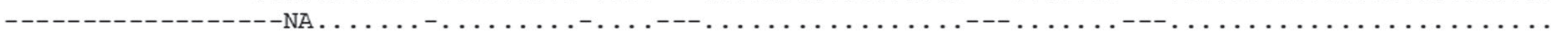
$201 \quad$ ORF26in.A 251 ATGT-GTCATTTATG-GGGCGCACATATCGTCTGGACGTAGACAACACGGA

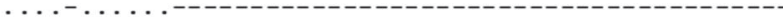

. C. .A.C. .A. .-----------

..........A-...A.TT.G

$\ldots \ldots \ldots \ldots \ldots \ldots \ldots \ldots \ldots \ldots$ TG.ATGT.

.TGT........................

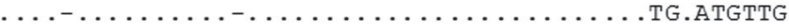

10RF72in.S

100

TTGCCCGC-СTCTATTATCAGCGCTGCAGGCTGTGCGCTGTTGGTTCCTGCCAACGTCATTCCGCAGGATACCCACTCGGGTGGGGTAGTTCCTCAGCTG

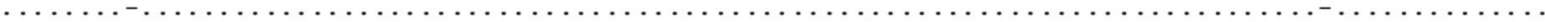

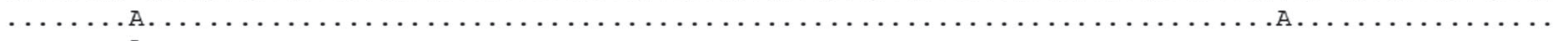

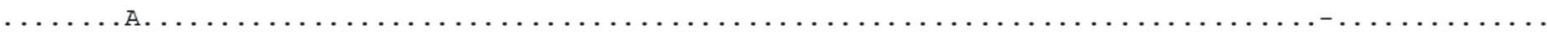

U $40667 * \quad \begin{aligned} & 101 \\ & \text { GCAAGCATATTGGGATGCGATGTTPCCGTTCTACAGGCG }\end{aligned}$

KS2 $4-\ldots \ldots \ldots$ GA---

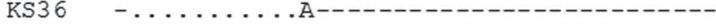

KS48

........GATC-

Figure 1. Panel A, Kaposi's sarcoma-associated herpesvirus (KSHV) detection by PCR in Kaposi's sarcoma lesions (KS) and histologically normal skin samples (NS). BCBL-1 is the positive control (DNA extracted from a KSHV-positive primary effusion lymphoma cell line), whereas 1st No and 2nd No are PCR assays without sample DNA ("No DNA" negative controls). ORF26 nested-PCR (ORF26in) was performed with $0.5 \mu \mathrm{L}$ of the respective PCR product from the previous KS330 PCR. Only KS28, KS36 and KS48 were positive for KSHV in KS330 PCR. However, all KS samples, but no NS sample, were found to be positive for KSHV after ORF26 in nested-PCR. In addition, samples KS20, KS24, KS28, KS36, KS48, and no NS sample were positive for KSHV by ORF72 PCR. Panels $B$ and $C$, PCR products of selected samples were submitted to automated nucleic acid sequencing, and the sequences were aligned with reference KSHV sequences from GenBank. In panel B, amplicons for KS330 (BCBL-1, KS36 and KS48) and ORF26in (KS20, KS22 and KS24) KSHV-positive samples were aligned with a segment comprising nucleotides 987-1219 from KSHV sequence accession \#U18551. In panel C, amplicons for ORF72 KSHV-positive samples (KS24, KS36 and KS48) were aligned with a segment comprising nucleotides 2225-2362 from the KSHV sequence accession \#U40667. Differences in nucleotide sequences are indicated. Annealing regions for outer and inner PCR primers are indicated by shaded and white boxes, respectively. 
coded by KSHV ORF16) and viral FLIP (FLICE inhibitory protein, ORF-K13) have anti-apoptotic properties, which may contribute to a poor prognosis in KS. The KSHV G-protein-coupled receptor can induce VEGF-mediated tumor growth, and viral cyclin-D (ORF72) may induce cell proliferation through $\mathrm{pRB}$ inactivation (30).

Using different techniques, KSHV infection has been detected in 80 to $100 \%$ of KS cases worldwide. Serologic studies detecting antibodies against KSHV latency-associated nuclear antigens have allowed viral detection in $80 \%$ of HIV-positive patients with KS, and in almost $2 \%$ of healthy blood donors from the United States (31). Interestingly, another antigen-based assay showed an even higher frequency of KSHV infection in the general population, i.e., $25 \%$ of randomly selected individuals were positive (20).

PCR-based procedures have proved to be reliable methods to evaluate KSHV infection in biological samples. Using this technique, Moore and Chang (22) detected the KSHV genome in frozen tissues from 11 cases of AIDS-associated KS (91\%), and all cases of classical KS $(\mathrm{N}=6)$, as well as HIVnegative homosexual patients with $\mathrm{KS}(\mathrm{N}=$
4). Similar results were obtained by Jin et al. (32), i.e., KSHV was detected in $100 \%$ of 17 KS biopsies, 12 from HIV-negative and 5 from HIV-positive patients. Combining PCR and in situ hybridization, Huang et al. (33) demonstrated KSHV infection in all KS biopsies from 12 HIV-positive and 2 HIVnegative patients, and the KSHV viral genome was also detected in $25 \%$ of semen and blood samples from HIV-positive individuals with $\mathrm{KS}$.

These data indicate the high sensitivity of the PCR method for the detection of KSHV. However, in a study by Pan and coworkers (28), the authors concluded that KSHV detection by PCR may involve a high rate of false-negative results. For this reason, optimization of $\mathrm{PCR}$ procedures is mandatory, and nested-PCR approaches as well as amplification of different segments of the KSHV genome are recommended in order to obtain accurate results (28). Using a standard PCR procedure to detect the KSHV genome in DNA from formalin-fixed paraffinembedded tissue from few cases, Caterinode-Araujo et al. (25) initially found the virus in 3/7 (42.9\%) KS cases; however, all of their samples were found to be positive after nested-PCR.

Table 1. Characteristics of Kaposi's sarcoma (KS) patients in the present study.

\begin{tabular}{|c|c|c|c|c|c|c|c|c|c|c|}
\hline \multirow[t]{2}{*}{$\mathrm{KS}$ forms } & \multirow[t]{2}{*}{$\mathrm{M} / \mathrm{F}$ ratio } & \multicolumn{2}{|c|}{ Stage of disease } & \multicolumn{6}{|c|}{ Age (years) } & \multirow[t]{2}{*}{ KSHV-positivity } \\
\hline & & & & Mean \pm SD & $\begin{array}{c}\text { Coefficient of } \\
\text { variation }\end{array}$ & Median & $\mathrm{P}_{25}$ & $P_{75}$ & Range & \\
\hline \multirow{5}{*}{$\begin{array}{l}\text { AIDS-associated } \\
(\mathrm{N}=29)\end{array}$} & $28: 1$ & Patch & 9/29 (31\%) & $36.9 \pm 11.3$ & $30.6 \%$ & 32 & 31.5 & 42.8 & $24-62$ & $88.9 \%$ \\
\hline & & Plaque & $9 / 29(31 \%)$ & $36.3 \pm 4.9$ & $13.5 \%$ & 36.5 & 32.5 & 40.0 & $29-43$ & $100 \%$ \\
\hline & & Nodular & $4 / 29(13.8 \%)^{*}$ & $36.8 \pm 14.9$ & $40.5 \%$ & 30 & 28.5 & 45.0 & $28-59$ & $100 \%$ \\
\hline & & Visceral & $6 / 29(20.7 \%)$ & $34.2 \pm 13.6$ & $39.8 \%$ & 39 & 22.0 & 45.5 & $16-47$ & $100 \%$ \\
\hline & & Inconclusive & $1 / 29(3.5 \%)$ & 53.0 & - & - & - & - & - & $100 \%$ \\
\hline \multirow{3}{*}{$\begin{array}{l}\text { Classical } \\
(N=20)\end{array}$} & $4: 1$ & Patch & $2 / 20(10 \%)$ & $49.0 \pm 28.3$ & $57.8 \%$ & 49 & 29.0 & 69.0 & $29-69$ & $100 \%$ \\
\hline & & Plaque & $3 / 20(15 \%)$ & $62.3 \pm 16.1$ & $25.8 \%$ & 69 & 50.3 & 72.8 & $44-74$ & $100 \%$ \\
\hline & & Nodular & $15 / 20(75 \%)^{\star}$ & $65.7 \pm 14.7$ & $22.4 \%$ & 72 & 49.8 & 75.0 & $41-85$ & $86.7 \%$ \\
\hline $\begin{array}{l}\text { latrogenic } \\
(N=2)\end{array}$ & $1: 1$ & Plaque & $2 / 2(100 \%)$ & $41.5 \pm 29.0$ & $69.9 \%$ & 41.5 & 21.0 & 62.0 & $21-62$ & $100 \%$ \\
\hline
\end{tabular}

Data are reported as mean $\pm S D . P_{25}, P_{75}=25$ th and 75 th percentiles, respectively. KSHV $=$ Kaposi's sarcoma-associated herpesvirus. ${ }^{*} \mathrm{P}<0.05$ between the indicated groups (Goodman's proportion test). 
In the present study, we detected 31/51 $(60.8 \%)$ KSHV-positive cases in the first PCR round. Fourteen of 20 initially negative cases $(70 \%)$ proved to be KSHV-positive with further analysis by nested-PCR. Additionally, 3 of the $6(50 \%)$ remaining cases had demonstrable KSHV ORF72 amplification. Overall, KSHV infection was detected by PCR in 48/51 (94.1\%) KS cases. These data emphasize the need for an optimized PCR procedure to minimize false-negative results, which may occur due to small quantity or high fragmentation of DNA extracted from formalin-fixed paraffin-embedded tissues, as well as a low viral burden in tissue biopsies and tumor scarcity in biopsy samples (e.g., early stage lesions of KS).

Some studies about KSHV transmission have demonstrated that the virus can be transmitted to transplanted patients by KSHVinfected donors (34). In this case, tumor development is associated with immunosuppressive therapy (35). Vertical KSHV transmission was demonstrated by Mantina et al. (36) who found viral DNA in two neonates; moreover, the authors emphasize that it may not be possible to determine if infection occurred in utero or intrapartum, but postnatal infection was ruled out due to the impossibility to detect DNA during the immediate postnatal period.

It was suggested that the sexual transmission of KSHV among homosexual men probably occurred because of anal receptive intercourse (37). A possible source of nonsexual horizontal transmission (e.g., through saliva) was suggested by a study that analyzed KSHV transmission in children before puberty (38). KSHV transmission by injection drug use was rare among drug users in Amsterdam (39), but another study demonstrated that KSHV seropositivity increased in association with injection drug use (40).

Few studies are available about KSHV frequency among KS patients in Brazil (2427). Keller et al. (26) evaluated 66 blood samples from HIV-positive patients, and
KSHV infection was detected by PCR in 29/ 39 (74.4\%) of KS-positive and 1/27 (3.7\%) of KS-negative cases. In serologic analysis, $38 / 39(97.4 \%)$ of KS-positive individuals had antibodies to viral antigen and 7/27 (26\%) of KS-negative individuals were positive to $\mathrm{KSHV}$ in at least one serologic analysis. They investigated immunodeficiency of HIV-positive patients through CD4 counts and observed a decrease in CD4 cells in HIV-positive patients with KS in the aggressive stage (26).

Analyzing 267 Brazilian patients, Zhang et al. (27) found anti-KSHV antibodies in 64-71\% HIV-positive patients with KS. In a more recent study, Biggar et al. (24) detected a new KSHV subtype (namely subtype E) in different Brazilian Amerindians from the Northern region of Brazil using an immunofluorescence approach. The authors observed that KSHV infection was endemic in this particular group and were unable to identify KSHV-associated diseases, such as KS or HIV infection. They used 746 samples from 16 different tribes and detected a prevalence of KSHV infection ranging from 0 to $100 \%$ (overall, a 53\% prevalence). KSHV immunofluorescence-positive cases were analyzed by quantitative PCR, and the viral genome was detected in 3/19 (16\%) of samples from peripheral blood mononuclear cells and in 1/16 (6.3\%) from saliva. It seems that oral transmission, rather than sexual transmission, was more important in this population since people with KSHV infection were $41 \%$ of the children (at least 10 years old) and $65 \%$ of the adults (30 years old) (24).

We could not find another study in the literature evaluating retrospectively KSHV infection in a larger number of KS biopsies than analyzed in the present study. Our results emphasize that KSHV is positively associated with all forms of KS and can be readily detected in tissue biopsies. Although PCR is extremely sensitive for detecting the KSHV genome in DNA samples from paraf- 
fin-embedded tissues, the optimization of PCR-based procedures is necessary in order to minimize false-negative results.

\section{Acknowledgments}

The authors are indebted to Diagnostika Laboratory, Consultoria em Patologia Labo- ratory, and to Dr. Luiz Alberto Veronese for providing additional Kaposi's sarcoma cases for this study. Additionally, we would like to thank Dr. Adalberto José Crocci for support with the statistical analysis, and Ms. Celene Gandin and Mr. Marcos Roberto Franchi for valuable technical support.

\section{References}

1. Kaposi M (1872). Idiopathisches multiples Pigmentsarkom der Haut. Archiv für Dermatologie und Syphilis, 4: 265-272.

2. Schwartz RA (2004). Kaposi's sarcoma: an update. Journal of Surgical Oncology, 87: 146-151.

3. Friedman-Kien AE \& Saltzman BR (1990). Clinical manifestations of classical, endemic African, and epidemic AIDS-associated Kaposi's sarcoma. Journal of the American Academy of Dermatology, 22: 1237-1250.

4. Kempf W \& Adams V (1996). Viruses in the pathogenesis of Kaposi's sarcoma - a review. Biochemical and Molecular Medicine, 58: 1-12.

5. Antman K \& Chang Y (2000). Kaposi's sarcoma. New England Journal of Medicine, 342: 1027-1038.

6. Beral V, Peterman TA, Berkelman RL et al. (1990). Kaposi's sarcoma among persons with AIDS: a sexually transmitted infection? Lancet, 335: 123-128.

7. Chang Y, Cesarman E, Pessin MS et al. (1994). Identification of herpesvirus-like DNA sequences in AIDS-associated Kaposi's sarcoma. Science, 266: 1865-1869.

8. Cesarman E, Chang Y, Moore PS et al. (1995). Kaposi's sarcomaassociated herpesvirus-like DNA sequences in AIDS-related bodycavity-based lymphomas. New England Journal of Medicine, 332: 1186-1191.

9. Cesarman E \& Knowles DM (1999). The role of Kaposi's sarcomaassociated herpesvirus (KSHV/HHV-8) in lymphoproliferative diseases. Seminars in Cancer Biology, 9: 165-174.

10. Huh J, Kang GH, Gong G et al. (1998). Kaposi's sarcoma-associated herpesvirus in Kikuchi's disease. Human Pathology, 29: 10911096.

11. Soulier J, Grollet L, Oksenhendler E et al. (1995). Kaposi's sarcoma-associated herpesvirus-like DNA sequences in multicentric Castleman's disease. Blood, 86: 176-180.

12. Aoki $Y$, Jaffe ES, Chang $Y$ et al. (1999). Angiogenesis and hematopoiesis induced by Kaposi's sarcoma-associated herpesvirus-encoded interleukin-6. Blood, 93: 4034-4043.

13. Ascherl G, Hohenadl C, Monini P et al. (1999). Expression of human herpesvirus-8 (HHV-8) encoded pathogenic genes in Kaposi's sarcoma (KS) primary lesions. Advances in Enzyme Regulation, 39: 331-339.

14. Cesarman E, Nador RG, Bai $F$ et al. (1996). Kaposi's sarcomaassociated herpesvirus contains $G$ protein-coupled receptor and cyclin D homologs which are expressed in Kaposi's sarcoma and malignant lymphoma. Journal of Virology, 70: 8218-8223.

15. Meinl E, Fickenscher $H$, Thome $M$ et al. (1998). Anti-apoptotic strategies of lymphotropic viruses. Immunology Today, 19: 474-479.

16. Rivas C, Thlick AE, Parravicini $C$ et al. (2001). Kaposi's sarcomaassociated herpesvirus LANA2 is a B-cell-specific latent viral pro- tein that inhibits p53. Journal of Virology, 75: 429-438.

17. Stürzl M, Blasig C, Schreier A et al. (1997). Expression of HHV-8 latency-associated T0.7 RNA in spindle cells and endothelial cells of AIDS-associated, classical and African Kaposi's sarcoma. International Journal of Cancer, 72: 68-71.

18. Stürzl M, Wunderlich A, Ascherl G et al. (1999). Human herpesvirus8 (HHV-8) gene expression in Kaposi's sarcoma (KS) primary lesions: an in situ hybridization study. Leukemia, 13: S110-S112.

19. Blasig C, Zietz C, Haar B et al. (1997). Monocytes in Kaposi's sarcoma lesion are productively infected by human herpesvirus 8 . Journal of Virology, 71: 7963-7968.

20. Lennette ET, Blackbourn DJ \& Levy JA (1996). Antibodies to human herpesvirus type 8 in the general population and in Kaposi's sarcoma patients. Lancet, 348: 858-861.

21. Whitby D, Howard MR, Tenant-Flowers M et al. (1995). Detection of Kaposi sarcoma associated herpesvirus in peripheral blood of HIVinfected individuals and progression to Kaposi's sarcoma. Lancet, 346: 799-802

22. Moore PS \& Chang $Y$ (1995). Detection of herpesvirus-like DNA sequences in Kaposi's sarcoma in patients with and without HIV infection. New England Journal of Medicine, 332: 1181-1185.

23. Dictor M, Rambech E, Way D et al. (1996). Human herpesvirus 8 (Kaposi's sarcoma-associated herpesvirus) DNA in Kaposi's sarcoma lesion, AIDS Kaposi's sarcoma cell lines, endothelial Kaposi's sarcoma simulators, and the skin of immunosuppressed patients. American Journal of Pathology, 148: 2009-2016.

24. Biggar Rj, Whitby D, Marshall V et al. (2000). Human herpesvirus 8 in Brazilian Amerindians: a hyperendemic population with a new subtype. Journal of Infectious Diseases, 181: 1562-1568.

25. Caterino-de-Araujo A, Calabrò ML, Favero A et al. (1997). Detection of herpes virus (KSHV) DNA sequences in Brazilian patients with AIDS-associated Kaposi's sarcoma. Brazilian Journal of Infectious Diseases, 1: 256-259.

26. Keller R, Zago A, Viana MC et al. (2001). HHV-8 infection in patients with AIDS-related Kaposi's sarcoma in Brazil. Brazilian Journal of Medical and Biological Research, 34: 879-886.

27. Zhang X, Fitzpatrick L, Campbell TB et al. (1998). Comparison of the prevalence of antibodies to human herpesvirus 8 (Kaposi's sarcoma-associated herpesvirus) in Brazil and Colorado. Journal of Infectious Diseases, 178: 1488-1491.

28. Pan L, Milligan L, Michaeli J et al. (2001). Polymerase chain reaction detection of Kaposi's sarcoma-associated herpesvirus-optimized protocols and their application to myeloma. Journal of Molecular Diagnostics, 3: 32-38.

29. Greer CE, Peterson SL, Kiviat NB et al. (1991). PCR amplification from paraffin-embedded tissues. Effects of fixative and fixation time. 
American Journal of Clinical Pathology, 95: 117-124.

30. Foreman KE (2001). Kaposi's sarcoma: the role of HHV-8 and HIV1 in pathogenesis. Expert Reviews in Molecular Medicine, 26: 1-17.

31. Whelan P \& Scadden DT (2000). New developments in the etiopathogenesis and treatment of HIV-related Kaposi's sarcoma. Clinical Dermatology, 18: 469-477.

32. Jin YT, Tsai ST, Yan JJ et al. (1996). Detection of Kaposi's sarcomaassociated herpesvirus-like DNA sequence in vascular lesions. American Journal of Clinical Pathology, 105: 360-363.

33. Huang YQ, Li JJ, Poiesz BJ et al. (1997). Detection of the herpesvirus-like DNA sequences in matched specimens of semen and blood from patients with AIDS-related Kaposi's sarcoma by polymerase chain reaction in situ hybridization. American Journal of Pathology, 150: 147-153.

34. Luppi M, Barozzi P, Santagostino G et al. (2000). Molecular evidence of organ-related transmission of Kaposi sarcoma-associated herpesvirus or human herpesvirus-8 in transplant patients. Blood, 96: 3279-3281.

35. Hengge UR, Ruzicka T, Tyring SK et al. (2002). Update on Kaposi's sarcoma and other HHV-8 associated diseases. Part 1: epidemiol- ogy, environmental predispositions, clinical manifestations, and therapy. Lancet, 2: 281-292.

36. Mantina H, Kankasa C, Klaskala W et al. (2001). Vertical transmission of Kaposi's sarcoma-associated herpesvirus. International Journal of Cancer, 94: 749-752.

37. Melbye M, Cook PM, Hjalgrim $\mathrm{H}$ et al. (1998). Risk factors for Kaposi's-sarcoma-associated herpesvirus (KSHV/HHV-8) seropositivity in a cohort of homosexual men, 1981-1996. International Journal of Cancer, 77: 543-548.

38. Mayama S, Cuevas LE, Sheldon J et al. (1998). Prevalence and transmission of Kaposi's sarcoma-associated herpesvirus (human herpesvirus 8) in Ugandan children and adolescents. International Journal of Cancer, 77: 817-820.

39. Renwick N, Dukers NHTM, Weverling GJ et al. (2002). Risk factors for human herpesvirus 8 infection in a cohort of drug users in the Netherlands, 1985-1996. Journal of Infectious Diseases, 185: 18081812.

40. Ablashi DV, Chatlynne LG, Whitman Jr JE et al. (2002). Spectrum of Kaposi's sarcoma-associated herpesvirus, or human herpesvirus 8 , disease. Clinical Microbiology Reviews, 15: 439-464. 